\title{
Novel dental adhesive containing antibacterial agents and calcium phosphate nanoparticles
}

\author{
Mary Anne S. Melo, ${ }^{1,2}$ Lei Cheng, ${ }^{1,3}$ Michael D. Weir, ${ }^{1}$ Ru-Ching Hsia, ${ }^{4}$ \\ Lidiany K. A. Rodrigues, ${ }^{2}$ Hockin H. K. Xu ${ }^{1,5,6,7}$
}

${ }^{1}$ Biomaterials and Tissue Engineering Division, Department of Endodontics, Prosthodontics and Operative Dentistry, University of Maryland School of Dentistry, Baltimore, Maryland 21201

${ }^{2}$ Faculty of Pharmacy, Dentistry and Nursing, Federal University of Ceara, Fortaleza, CE, Brazil

${ }^{3}$ State Key Laboratory of Oral Diseases, West China School of Stomatology, Sichuan University, Chengdu, China

${ }^{4}$ Electron Microscopy Core Facility, University of Maryland School of Dentistry, Baltimore, Maryland 21201

${ }^{5}$ Center for Stem Cell Biology and Regenerative Medicine, University of Maryland School of Medicine,

Baltimore, Maryland 21201

${ }^{6}$ Marlene and Stewart Greenebaum Cancer Center, University of Maryland School of Medicine, Baltimore, Maryland 21201

${ }^{7}$ Department of Mechanical Engineering, University of Maryland, Baltimore County, Maryland 21250

Received 15 July 2012; revised 11 October 2012; accepted 11 November 2012

Published online 20 December 2012 in Wiley Online Library (wileyonlinelibrary.com). DOI: 10.1002/jbm.b.32864

\begin{abstract}
Secondary caries remains the main reason for dental restoration failure. Replacement of failed restorations accounts for $50 \%-70 \%$ of all restorations performed. Antibacterial adhesives could inhibit biofilm acids at tooth-restoration margins, and calcium phosphate (CaP) ions could remineralize tooth lesions. The objectives of this study were to: (1) incorporate nanoparticles of silver (NAg), quaternary ammonium dimethacrylate (QADM), and nanoparticles of amorphous calcium phosphate (NACP) into bonding agent; and (2) investigate their effects on dentin bonding and microcosm biofilms. An experimental primer was made with pyromellitic glycerol dimethacrylate (PMGDM) and 2-hydroxyethyl methacrylate (HEMA). An adhesive was made with bisphenol-A-glycerolate dimethacrylate (BisGMA) and triethylene glycol dimethacrylate (TEGDMA). NAg was incorporated into primer at $0.1 \mathrm{wt} \%$. The adhesive contained $0.1 \% \mathrm{NAg}$ and $10 \%$ QADM, and $0 \%-$ $40 \%$ NACP. Incorporating NAg into primer and NAg-QADMNACP into adhesive did not adversely affect dentin bond strength $(p>0.1)$. Scanning electron microscopy showed
\end{abstract}

numerous resin tags, and transmission electron microscopy revealed NAg and NACP in dentinal tubules. Viability of human saliva microcosm biofilms on primer/adhesive/composite disks was substantially reduced via NAg and QADM. Metabolic activity, lactic acid, and colony-forming units of biofilms were much lower on the new bonding agents than control $(p<0.05)$. In conclusion, novel dental bonding agents containing NAg, QADM, and NACP were developed with the potential to kill residual bacteria in the tooth cavity and inhibit the invading bacteria along tooth-restoration margins, with NACP to remineralize tooth lesions. The novel method of combining antibacterial agents (NAg and QADM) with remineralizing agent (NACP) may have wide applicability to other adhesives for caries inhibition. (c) 2012 Wiley Periodicals, Inc. J Biomed Mater Res Part B: Appl Biomater 101B: 620-629, 2013.

Key Words: antibacterial primer and adhesive, dentin bond strength, silver nanoparticles, calcium phosphate nanoparticles, human saliva microcosm biofilm, caries inhibition

How to cite this article: Melo Mary Anne S., Cheng L, Weir MD, Hsia R-C, Rodrigues Lidiany K. A., Xu Hockin H. K.. 2013. Novel dental adhesive containing antibacterial agents and calcium phosphate nanoparticles. J Biomed Mater Res Part B 2013:101B:620629.

\section{INTRODUCTION}

Resin composites are being increasingly used as esthetic dental restorations. ${ }^{1-3}$ Their popularity is a result of significant improvements in properties and performance. ${ }^{4-7}$ Nonetheless, composites tend to accumulate more biofilms in vivo, ${ }^{8,9}$ which could produce acids and cause dental caries. ${ }^{10,11}$ Recurrent caries is the main reason for restora- tion failure, and replacement of the failed restorations accounts for $50 \%-70 \%$ of all operative work. ${ }^{12-14}$ To reduce secondary (recurrent) caries, novel antibacterial resins containing quaternary ammonium salts (QAS) were developed. ${ }^{15-20}$ Resins containing 12-methacryloyloxydodecylpyridinium bromide (MDPB) significantly reduced the bacteria growth. ${ }^{15,16,21}$ Other resins used antibacterial agents such as

Correspondence to: H. H. K. Xu; e-mail: hxu@umaryland.edu or L. K. A. Rodrigues; e-mail: lidianykarla@yahoo.com Contract grant sponsor: NIH; contract grant numbers: R01DE17974, R01DE14190

Contract grant sponsor: Coordination for Improvement of Higher Education/Fulbright Doctoral Program; contract grant number: BEX0523/11-9 Contract grant sponsor: National Natural Science Foundation of China; contract grant number: 81100745

Contract grant sponsor: University of Maryland School of Dentistry 
methacryloxylethyl cetyl dimethyl ammonium chloride (DMAE-CB) and cetylpyridinium chloride (CPC). ${ }^{17,18,22,23}$

Another method to combat dental caries involved the development of antibacterial resins by incorporating silver (Ag) filler particles. ${ }^{24-26}$ Nanoparticles of Ag (NAg) were demonstrated to be effective for antibacterial applications. ${ }^{20,26,27} \mathrm{~A}$ different method for caries-inhibition used calcium phosphate (CaP) particle-filled composites, which released $\mathrm{Ca}$ and $\mathrm{P}$ ions and remineralized tooth lesions. ${ }^{28-30}$ Recently, CaP nanoparticles were synthesized via a spray-drying technique. ${ }^{31}$ The new nanocomposites containing nanoparticles of amorphous calcium phosphate (NACP) released $\mathrm{Ca}$ and $\mathrm{P}$ ions similar to traditional $\mathrm{CaP}$ composites, while possessing much higher mechanical properties for load-bearing tooth restorations. ${ }^{32,33}$

Besides composites, it is also important to develop caries-inhibiting adhesives. Adhesive bonds the composite to the tooth structure and infiltrates into dentinal tubules. ${ }^{34-36}$ Extensive studies were performed to improve and characterize enamel and dentin bonding. ${ }^{37-40}$ Residual bacteria could exist in the prepared tooth cavity, and microleakage could allow bacteria to invade the tooth-restoration interfaces. ${ }^{15,22}$ Therefore, antibacterial adhesives were developed using MDPB and other antibacterial agents. ${ }^{21-23}$ It is also beneficial to render the primer antibacterial because it directly contacts tooth structure. ${ }^{41,42}$ Recently, quaternary ammonium dimethacrylate (QADM) was combined with $\mathrm{NAg}$ in a commercial primer (SBMP, 3M) ${ }^{43}$ However, QADM and NAg have not been incorporated into any other adhesives. It remains to be investigated (1) whether QADM and NAg could be incorporated into another bonding system to also impart potent antibacterial functions; (2) whether NACP (as a remineralizing agent with $\mathrm{Ca}$ and $\mathrm{P}$ ions) can be incorporated into the antibacterial adhesive without compromising the dentin bond strength; (3) whether the NACP and NAg nanoparticles could flow into the dentinal tubules with the adhesive. The rationale was that while QADM and NAg could eradicate residual bacteria in the tooth cavity and dentinal tubules, NACP could remineralize the remnants of lesions in the tooth cavity, thus yielding a unique combination of antibacterial/remineralizing capabilities.

Accordingly, the objectives of this study were to incorporate NAg, QADM, and NACP into an experimental bonding system and to investigate the effects on anti-biofilm and dentin bonding properties. It was hypothesized that: (1) The new experimental bonding agent containing NAg and QADM would greatly reduce the biofilm viability, metabolic activity, and lactic acid production in a dental plaque microcosm biofilm model; (2) Incorporation of NACP into the experimental bonding agent containing NAg and QADM would not decrease the dentin bond strength; (3) NAg and NACP could flow with the bonding agent into the dentinal tubules.

\section{MATERIALS AND METHODS}

\section{Synthesis of experimental bonding agent containing NAg, QADM, and NACP}

The experimental primer contained pyromellitic glycerol dimethacrylate (PMGDM) (Hampford, Stratford, CT) and 2-hydroxyethyl methacrylate (HEMA) (Esstech, Essington, PA) at a mass ratio 3.3/1, with $50 \%$ acetone solvent (all mass fractions). ${ }^{44}$ The photo-initiator for the primer and adhesive was $1 \%$ phenyl bis(2,4,6-trimethylbenzoyl) phosphine oxide (BAPO) (Irgacure819, Ciba Chemicals, Japan). The experimental adhesive consisted of bisphenol A glycerolate dimethacrylate (BisGMA) and triethylene glycol dimethacrylate (TEGDMA) (Esstech) at $70 / 30$ mass ratio. ${ }^{45}$

Silver 2-ethylhexanoate salt (Strem, New Buryport, MA) of $0.1 \mathrm{~g}$ was dissolved in $1 \mathrm{~g}$ of 2-(tert-butylamino)ethyl methacrylate (TBAEMA, Sigma). ${ }^{20,26}$ TBAEMA was used because it improves the solubility by forming $\mathrm{Ag}-\mathrm{N}$ coordination bonds with $\mathrm{Ag}$ ions, thereby facilitating the $\mathrm{Ag}$ salt to dissolve in the resin solution. TBAEMA was selected as it contains reactive methacrylate groups and therefore can be chemically incorporated into a dental resin on photopolymerization. ${ }^{26}$ This method produced NAg in situ in the resin matrix with particle sizes of less than $10 \mathrm{~nm} \cdot{ }^{20,26}$ This study used a silver 2-ethylhexanoate/(primer + silver 2-ethylhexanoate) mass fraction of $0.1 \%$, because this imparted a strong antibacterial effect, without adversely affecting dentin bond strength or primer color in preliminary studies. The adhesive contained a silver 2-ethylhexanoate/(adhesive + silver 2-ethylhexanoate) of $0.1 \%$.

In addition, QADM and NACP were incorporated into the adhesive. Bis(2-methacryloyloxy-ethyl) dimethylammonium bromide, a quaternary ammonium dimethacrylate (QADM), was recently synthesized. ${ }^{19}$ The synthesis of QADM was performed using a modified Menschutkin reaction, where a tertiary amine group was reacted with an organo-halide. A benefit of this reaction is that the reaction products are generated at virtually quantitative amounts and require minimal purification. ${ }^{19}$ Briefly, $10 \mathrm{mmol}$ of 2 - $(\mathrm{N}, \mathrm{N}$-dimethylamino)ethyl methacrylate (Sigma-Aldrich, St. Louis, MO) and 10 mmol of 2-bromoethyl methacrylate (Monomer-Polymer Laboratories, Trevose, PA) were combined in ethanol and stirred at $60^{\circ} \mathrm{C}$ for $24 \mathrm{~h}^{19,20}$ This yielded QADM as a clear, colorless, and viscous liquid, which was mixed with the adhesive at QADM/(adhesive + QADM) mass fraction of $10 \%$, following a previous study. ${ }^{43}$

A spray-drying technique was used to make NACP. ${ }^{31,32,46}$ Amorphous calcium phosphate (ACP, $\mathrm{Ca}_{3}\left[\mathrm{PO}_{4}\right]_{2}$ ) is important because it is a precursor that can convert to apatite, similar to the minerals in tooth enamel and dentin. A spraying solution was prepared by adding $1.5125 \mathrm{~g}$ of acetic acid glacial (J.T. Baker, Phillipsburg, NJ) into $500 \mathrm{~mL}$ of distilled water. Then, $0.8 \mathrm{~g}$ of calcium carbonate $\left(\mathrm{CaCO}_{3}\right.$, Fisher, Fair Lawn, $\mathrm{NJ})$ and $5.094 \mathrm{~g}$ of DCPA (Baker) were dissolved into the acetic acid solution. The final $\mathrm{Ca}$ and $\mathrm{PO}_{4}$ ionic concentrations were $8 \mathrm{mmol} / \mathrm{L}$ and $5.333 \mathrm{mmol} / \mathrm{L}$, respectively. This yielded a Ca/P molar ratio of 1.5 , the same as that for ACP. This solution was sprayed through a nozzle into a heated chamber. The water and volatile acid were evaporated into the dry, heated air flow, and expelled into an exhaust-hood. The dried particles were collected by an electrostatic precipitator. ${ }^{32}$ This method produced NACP with a mean particle size of $112 \mathrm{~nm}^{32}$ The NACP were mixed with the adhesive at $\mathrm{NACP} /(\mathrm{NACP}+$ adhesive) mass fractions of $0 \%, 10 \%, 20 \%$, $30 \%$, and $40 \%$, following previous studies. ${ }^{32,33}$ 
Therefore, seven primer and adhesive systems were formulated:

1. Experimental primer control and adhesive control (termed "P control, A control").

2. P+NAg, A control (Primer had 0.1\% NAg, while adhesive had no NAg).

3. $\mathrm{P}+\mathrm{NAg}, \mathrm{A}+\mathrm{NAg}+\mathrm{QADM}$ (Both $\mathrm{P}$ and $\mathrm{A}$ had $0.1 \% \mathrm{NAg}$. A also had $10 \%$ QADM).

4. $\mathrm{P}+\mathrm{NAg}, \mathrm{A}+\mathrm{NAg}+\mathrm{QADM}+10 \mathrm{NACP}$ (10NACP means $10 \%$ NACP by mass).

5. $\mathrm{P}+\mathrm{NAg}, \mathrm{A}+\mathrm{NAg}+\mathrm{QADM}+20 \mathrm{NACP}$.

6. $\mathrm{P}+\mathrm{NAg}, \mathrm{A}+\mathrm{NAg}+\mathrm{QADM}+30 \mathrm{NACP}$.

7. $\mathrm{P}+\mathrm{NAg}, \mathrm{A}+\mathrm{NAg}+\mathrm{QADM}+40 \mathrm{NACP}$.

Dentin bond strength and scanning electron microscopy and transmission electron microscopy examinations

The use of extracted human teeth was approved by the University of Maryland. Caries-free third-molars were ground to remove the occlusal enamel. ${ }^{43,47}$ Flat mid-coronal dentin surfaces of caries-free molars were prepared by cutting off the tips of crowns with a diamond saw (Isomet, Buehler, Lake Bluff, IL). Each tooth was embedded in a poly-carbonate holder (Bosworth, Skokie, IL) and ground perpendicularly to the longitudinal axis on 320-grit silicon carbide paper until occlusal enamel was removed. Dentin was etched with $37 \%$ phosphoric acid for $15 \mathrm{~s}{ }^{43,47}$ A primer was applied, then an adhesive was applied and light-cured for $10 \mathrm{~s}$ (Optilux VCL401, Demetron, Danbury, CT). A stainless-steel iris (inner diameter $=4 \mathrm{~mm}$, thickness $=$ $1.5 \mathrm{~mm}$ ) was held against the adhesive-treated dentin, and the opening was filled with a composite (TPH, Caulk/Dentsply, Milford, DE) and light-cured for $60 \mathrm{~s}$. The specimens were stored in water at $37^{\circ} \mathrm{C}$ for $24 \mathrm{~h}^{43,47}$ To measure the dentin shear bond strength, a chisel was aligned parallel to the compositedentin interface and loaded (MTS, Eden Prairie, MN) at 0.5 $\mathrm{mm} / \mathrm{min}$ until the composite-dentin bond failed. Dentin shear bond strength $S_{\mathrm{D}}=4 P /\left(\pi d^{2}\right)$, where $P$ is load-at-failure, and $d$ is composite diameter. ${ }^{43,47}$

To examine the dentin-adhesive interface, the bonded teeth were cut longitudinally. The sections were treated with $50 \%$ phosphoric acid and $10 \% \mathrm{NaOCl}^{43}$ then goldcoated and examined via scanning electron microscopy (SEM, Quanta 200, FEI, Hillsboro, OR). For transmission electron microscopy (TEM), thin sections with an approximate thickness of $120 \mu \mathrm{m}$ were cut and fixed with $2 \%$ paraformaldehyde and $2.5 \%$ glutaraldehyde following a previous study. ${ }^{48}$ Samples were embedded in epoxy (Spurr's, Electron Microscopy Sciences, PA). Ultra-thin sections with approximate thickness of $100 \mathrm{~nm}$ were cut using a diamond knife (Diatome, Bienne, Switzerland) with an ultra-microtome (EM-UC7, Leica, Germany). The non-demineralized sections were examined in TEM (Tecnai-T12, FEI).

\section{Dental plaque microcosm biofilm model and live/dead assay}

The microcosm biofilm model was approved by the University of Maryland. Human saliva was shown to be ideal for growing plaque microcosm biofilms in vitro, with the advantage of maintaining much of the complexity and heterogeneity of the dental plaque in vivo. ${ }^{49}$ The saliva for biofilm inoculums was collected from a healthy adult donor having natural dentition without active caries or periopathology, and without the use of antibiotics within the last 3 months, following a previous study. ${ }^{43}$ The donor did not brush teeth for $24 \mathrm{~h}$ and abstained from food/drink intake for at least $2 \mathrm{~h}$ before donating saliva. Stimulated saliva was collected during parafilm chewing and kept on ice. Saliva was diluted in sterile glycerol to a concentration of $70 \%$ saliva and $30 \%$ glycerol, and stored at $-80^{\circ} \mathrm{C}^{43}$

Layered disks were fabricated as schematically shown in Figure 3(A), following previous studies. ${ }^{41,43}$ A polyethylene mold with an inner diameter of $9 \mathrm{~mm}$ and a thickness of 2 mm was situated on a glass slide. A primer was first applied into the mold to cover the glass slide. After drying with a stream of air, the adhesive was applied and cured for $20 \mathrm{~s}$ with an Optilux curing unit (VCL 401, Demetron Kerr, Danbury, CT). Then, a composite (TPH) was placed on the adhesive to fill the disk mold and light-cured for $1 \mathrm{~min}$. The cured disks were removed from the molds, immersed in sterile water and agitated for $1 \mathrm{~h}$ to remove any uncured monomer, following a previous study. ${ }^{41}$ The disks were then dried and sterilized with ethylene oxide (Anprolene AN 74i, Andersen, Haw River, NC).

The saliva-glycerol stock was added, with 1:50 final dilution, to a growth medium as inoculum. The growth medium contained mucin (type II, porcine, gastric) at a concentration of $2.5 \mathrm{~g} / \mathrm{L}$; bacteriological peptone, $2.0 \mathrm{~g} / \mathrm{L}$; tryptone, $2.0 \mathrm{~g} / \mathrm{L}$; yeast extract, $1.0 \mathrm{~g} / \mathrm{L} ; \mathrm{NaCl}, 0.35 \mathrm{~g} / \mathrm{L}, \mathrm{KCl}, 0.2 \mathrm{~g} / \mathrm{L} ; \mathrm{CaCl}_{2}, 0.2$ $\mathrm{g} / \mathrm{L}$; cysteine hydrochloride, $0.1 \mathrm{~g} / \mathrm{L}$; haemin, $0.001 \mathrm{~g} / \mathrm{L}$; and vitamin $\mathrm{K} 1,0.0002 \mathrm{~g} / \mathrm{L}$, at $\mathrm{pH} 7 .^{50}$ The inoculum was cultured at $37^{\circ} \mathrm{C}$ in an incubator containing $5 \% \mathrm{CO}_{2}$ for $24 \mathrm{~h}$. Each disk was placed into a well of 24-well plates, with the primer surface on the top. To each well was added $1.5 \mathrm{~mL}$ of inoculum, which was incubated for $8 \mathrm{~h}$. The disks were transferred to new 24-well plates with fresh medium and incubated. After $16 \mathrm{~h}$, the disks were transferred to new 24-well plates with fresh medium and incubated for $24 \mathrm{~h}$. This constructed a 2-day incubation, which was shown to form dental plaque microcosm biofilms. ${ }^{43}$ The disks with 2-day biofilms were washed with phosphate buffered saline (PBS) and stained using a live/dead bacterial viability kit (Molecular Probes, Eugene, OR). Live bacteria were stained with Syto 9 to produce a green fluorescence, and bacteria with compromised membranes were stained with propidium iodide to produce a red fluorescence. The stained disks were examined using an epifluorescence microscope (TE2000-S, Nikon, Melville, NY). ${ }^{20}$

\section{Metabolic activity of microcosm biofilms}

The MTT (3-[4,5-Dimethylthiazol-2-yl]-2,5-diphenyltetrazolium bromide) assay is a colorimetric assay that measures the enzymatic reduction of MTT, a yellow tetrazole, to formazan. $^{20}$ Each disk with the 2-day biofilm was transferred to a new 24-well plate, then $1 \mathrm{~mL}$ of MTT dye $(0.5 \mathrm{mg} / \mathrm{mL}$ MTT in PBS) was added to each well and incubated at $37^{\circ} \mathrm{C}$ in $5 \% \mathrm{CO}_{2}$ for $1 \mathrm{~h}$. During this process, metabolically active 
bacteria reduced the MTT to purple formazan. After $1 \mathrm{~h}$, the disks were transferred to a new 24-well plate, $1 \mathrm{~mL}$ of dimethyl sulfoxide (DMSO) was added to solubilize the formazan crystals, and the plate was incubated for $20 \mathrm{~min}$ with gentle mixing at room temperature in the dark. After mixing via pipetting, $200 \mu \mathrm{L}$ of the DMSO solution from each well was transferred to a 96-well plate, and the absorbance at $540 \mathrm{~nm}$ (optical density $\mathrm{OD}_{540}$ ) was measured via a microplate reader (SpectraMax M5, Molecular Devices, Sunnvale, CA). A higher absorbance is related to a higher formazan concentration, which indicates a higher metabolic activity in the biofilm on the disk. ${ }^{20}$

\section{Lactic acid production and colony-forming units of microcosm biofilms}

To measure lactic acid production, disks with 2-day biofilms were transferred to 24-well plates containing buffered-peptone water (BPW) plus $0.2 \%$ sucrose, and incubated for $3 \mathrm{~h}$ to allow the biofilms to produce acid. The lactate concentrations in the BPW solutions were determined using an enzymatic (lactate dehydrogenase) method, following previous studies. $^{20,43}$ The microplate reader (SpectraMax M5) was used to measure the absorbance at $340 \mathrm{~nm}$ (optical density $\mathrm{OD}_{340}$ ) for the collected BPW solutions. Standard curves were prepared using a lactic acid standard (Supelco, Bellefonte, PA).

For colony-forming units (CFUs), disks with 2-day biofilms were transferred into tubes with $2 \mathrm{~mL}$ cysteine peptone water, and the biofilms were harvested by sonication and vortexing via a vortex mixer (Fisher, Pittsburgh, PA). Four types of agar plates were used. First, tryptic soy blood agar culture plates were used to determine total microorganisms. ${ }^{43}$ Second, mitis salivarius agar (MSA) culture plates, containing $15 \%$ sucrose, were used to determine total streptococci. ${ }^{51}$ This is because MSA contains selective agents crystal violet, potassium tellurite, and trypan blue, which inhibit most gram-negative bacilli and most grampositive bacteria except streptococci, thus enabling streptococci to grow. ${ }^{51}$ Third, cariogenic mutans streptococci are known to be resistant to bacitracin, and this property is often used to isolate mutans streptococci from the highly heterogeneous oral microflora. Hence, MSA agar culture plates plus 0.2 units of bacitracin per $\mathrm{mL}$ were used to determine mutans streptococci. ${ }^{52}$ Forth, Rogosa SL culture plates were used to determine lactobacilli. Rogosa SL plates contained high levels of sodium acetate and ammonium citrate at a low $\mathrm{pH}$ which would inhibit most microorganisms but allow the growth of lactobacilli. ${ }^{53}$

One-way analysis of variance was performed to detect the significant effects of the variables. Tukey's multiple comparison was used to compare the data at a $p$ value of 0.05 .

\section{RESULTS}

Figure 1 shows the dentin bonding results: (A) Dentin shear bond strengths (mean \pm sd; $n=10$ ), and (B) representative SEM image of dentin-adhesive interface. In (A), dentin shear bond strengths were similar between the control and those containing NAg, QADM, and NACP ( $p>0.1)$. Therefore, adding NAg, QADM, and NACP from $10 \%$ to $40 \%$ did not sig-
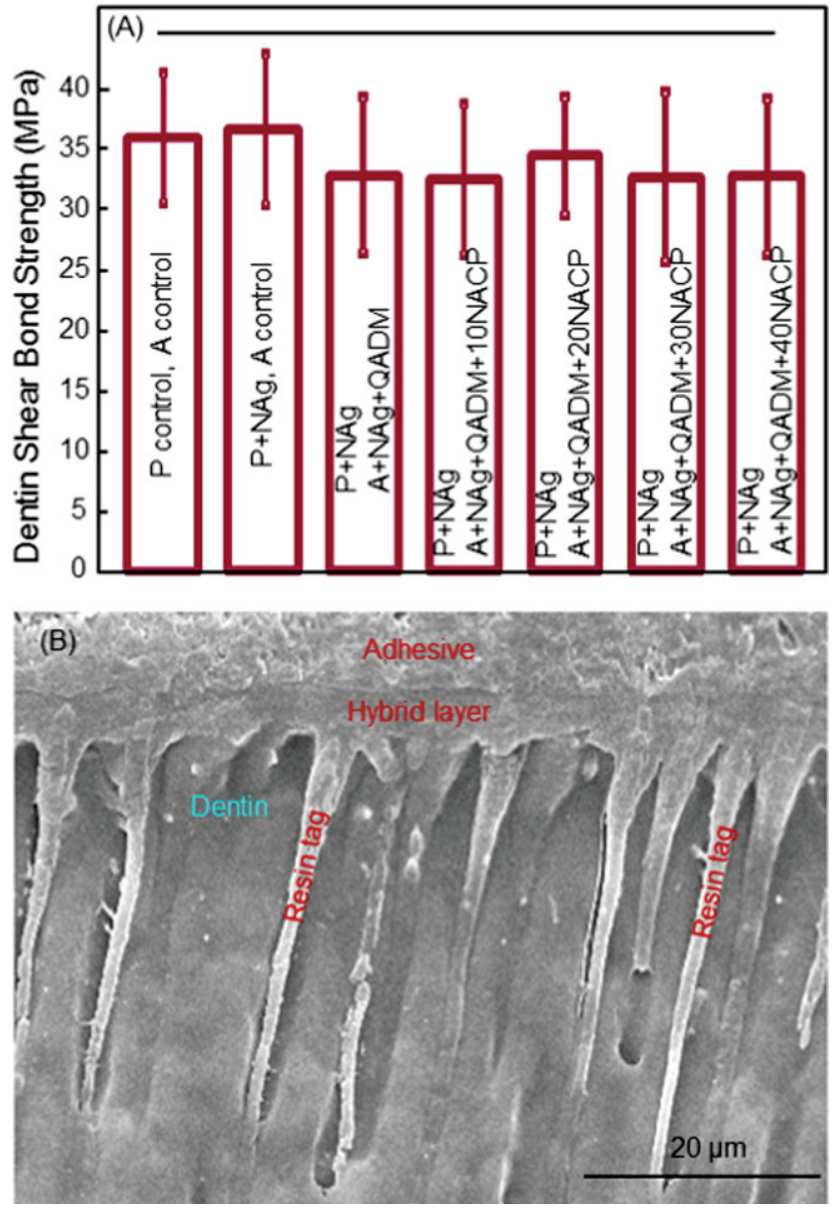

FIGURE 1. Dentin bond testing. (A) Dentin shear bond strength using extracted human molars. Each value is mean \pm sd $(n=10)$. $\mathrm{P}=$ primer. $\mathrm{A}=$ adhesive. $\mathrm{P}+\mathrm{NAg}$ had $0.1 \% \mathrm{NAg}$ (by mass) in primer. $\mathrm{A}+\mathrm{NAg}+\mathrm{QADM}$ had $0.1 \% \mathrm{NAg}+10 \%$ QADM in adhesive. The hybrid layer is the demineralized collagen of dentin infiltrated by primer and adhesive (hybrid layer $=$ collagen + primer + adhesive). Adding NAg, QADM, and NACP into the bonding agent did not decrease the dentin bond strength. Horizontal line indicates statistically similar values ( $p$ $>0.1$ ). (B) SEM examination of dentin-adhesive interfaces revealed numerous resin tags from well-filled dentinal tubules, with an example shown for the control. Other groups had similar dentin-adhesive interfacial features. [Color figure can be viewed in the online issue, which is available at wileyonlinelibrary.com.]

nificantly compromise the dentin bond strength, compared with the control. In (B), SEM examination revealed the formation of numerous resin tags from well-filled dentinal tubules. An example is shown for control [Figure 1(B)], while other groups had similar dentin-adhesive interfacial features.

Representative TEM images of the dentin-adhesive interface are shown in Figure 2. In (A), an example of a resin tag in a dentinal tubule shows that the NACP were successfully flowed with the adhesive resin into the dentinal tubule. This example was for the bonding system of $\mathrm{P}+\mathrm{NAg}, \mathrm{A}+\mathrm{NAg}+$ QADM+30NACP. An even higher magnification in (B) shows that the NAg was also incorporated into the dentinal tubules.

Figure 3 shows the live/dead assay results. The layered disk structure is schematically shown in (A). The 2-day biofilm adherent on the disk surface was stained, with the live 

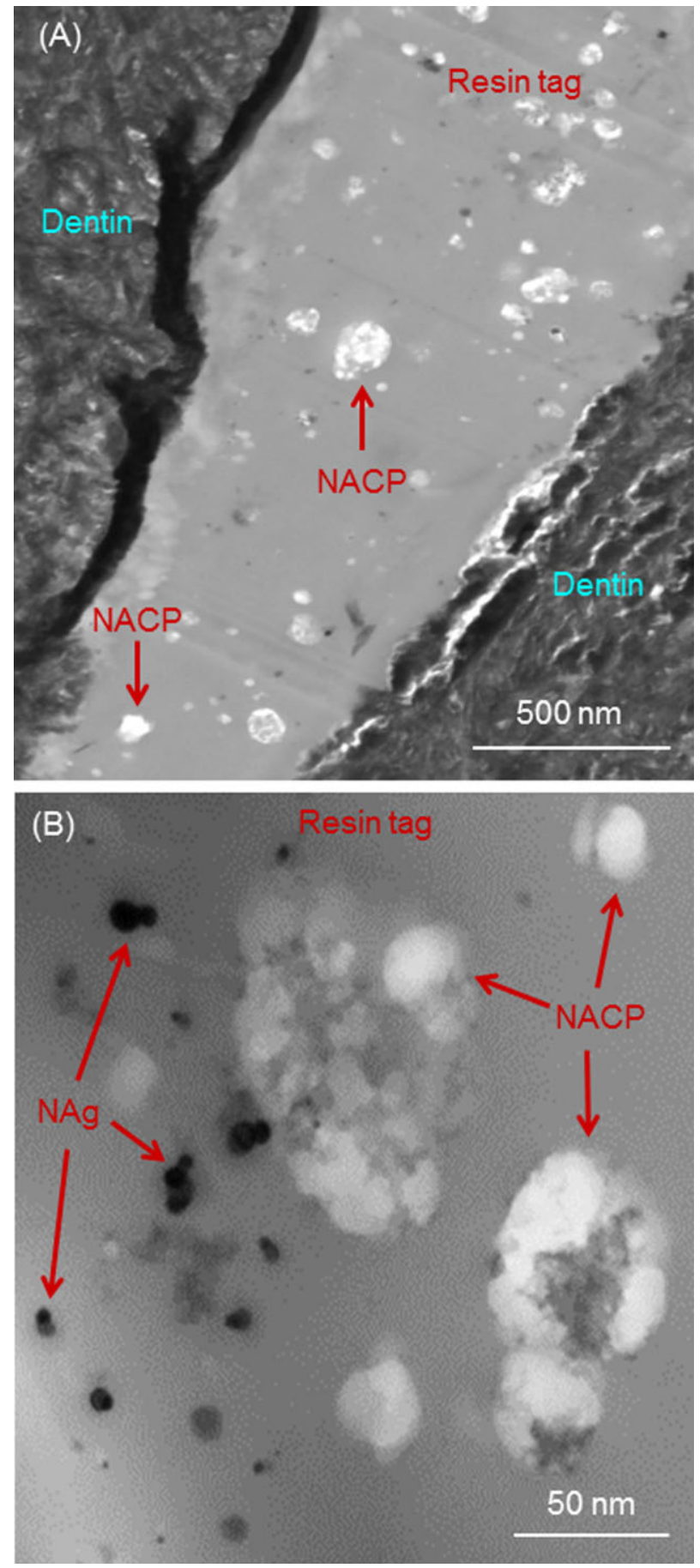

FIGURE 2. High magnification TEM examination of dentin-adhesive interfaces. (A) Typical resin tag for the primer and adhesive group of $\mathrm{P}+\mathrm{NAg}, \mathrm{A}+\mathrm{NAg}+\mathrm{QADM}+30 \mathrm{NACP}$, shown as an example. Arrows indicate NACP that successfully flowed with the adhesive into dentinal tubules. (B) Higher magnification TEM revealed NAg as well as $\mathrm{NACP}$ in the resin tags in the dentinal tubules. The NAg appeared as black dots in TEM images with sizes of less than $10 \mathrm{~nm}$, similar to those observed in previous study. ${ }^{49}$ [Color figure can be viewed in the online issue, which is available at wileyonlinelibrary.com.]

bacteria staining a green color, and the dead and compromised bacteria staining red. The control disks showed primarily live bacteria (B). Disks with NAg and NAg+QADM had much more dead bacteria (C and D). Adding 10\%-40\% NACP did not noticeably alter the biofilms ( $\mathrm{E}$ and $\mathrm{F}$ ).

The MTT assay of biofilms is plotted in Figure 4 (mean $\pm \mathrm{sd} ; n=6$ ). As expected, biofilms on the control disks had a relatively high metabolic activity. Incorporation of QADM and NAg greatly decreased the metabolic activity of the adherent biofilms ( $p<0.05$ ). Adding 10\% and 20\% NACP into the adhesive did not significantly change the metabolic activity, while $30 \%$ and $40 \%$ NACP significantly lowered the metabolic activity $(p<0.05)$.

Figure 5 plots the lactic acid production of biofilms (mean \pm sd; $n=6$ ). Biofilms on control disks secreted the most amount of lactic acid. Incorporation of NAg and QADM into the control bonding agent substantially reduced the lactic acid production $(p<0.05)$. Further adding NACP to the adhesive in general did not significantly affect the lactic acid production of the biofilms, except for the $40 \%$ NACP in the adhesive which had a significantly lower lactic acid $(p<$ 0.05).

Biofilm CFU counts are plotted in Figure 6 for: (A) Total microorganisms, (B) total streptococci, (C) mutans streptococci, and (D) lactobacilli (mean \pm sd; $n=6$ ). In each case, the control bonding agent had relatively high CFU, and the addition of NAg and QADM substantially reduced the CFU ( $p$ $<0.01$ ). In general, adding NACP from $10 \%$ to $40 \%$ into the adhesive did not significantly affect the CFU counts, except for total streptococci where the 40\% NACP group had a significantly lower CFU than that without NACP ( $p<$ 0.05). These results demonstrate that adding NAg and QADM into the experimental bonding agent was effective in reducing the microcosm biofilm viability and lactic acid production.

\section{DISCUSSION}

This study incorporated NAg into an experimental primer, and QADM and NACP into an experimental adhesive for the first time. A previous study incorporated NAg and QADM into a commercial bonding agent (SBMP, 3M), ${ }^{43}$ but it was unclear if the NAg and QADM could be similarly applied to other bonding systems to achieve potent antibacterial activities without compromising the dentin bond strength. This study used a primer comprising of PMGDM and HEMA, and an adhesive comprising of BisGMA and TEGDMA, which are an experimental bonding system and not commercially available. The results showed that QADM and NAg were successfully incorporated into this experimental bonding system, exhibiting strong antibacterial effects, which suggest that QADM and NAg are promising for applications into other bonding agents. Although several previous studies used single-species bacteria models, ${ }^{19,26,42}$ this study used a dental plaque microcosm model. Dental plaque is a complicated ecosystem with about 1000 bacterial species, ${ }^{11}$ hence microcosm models could maintain much of the complexity and heterogeneity in vivo. ${ }^{49}$ The new bonding agent reduced the biofilm viability and CFU to about one-third of those of the control. CFU counts of total microorganisms, total streptococci, mutans streptococci, and lactobacilli were all greatly reduced by the new bonding agents. 

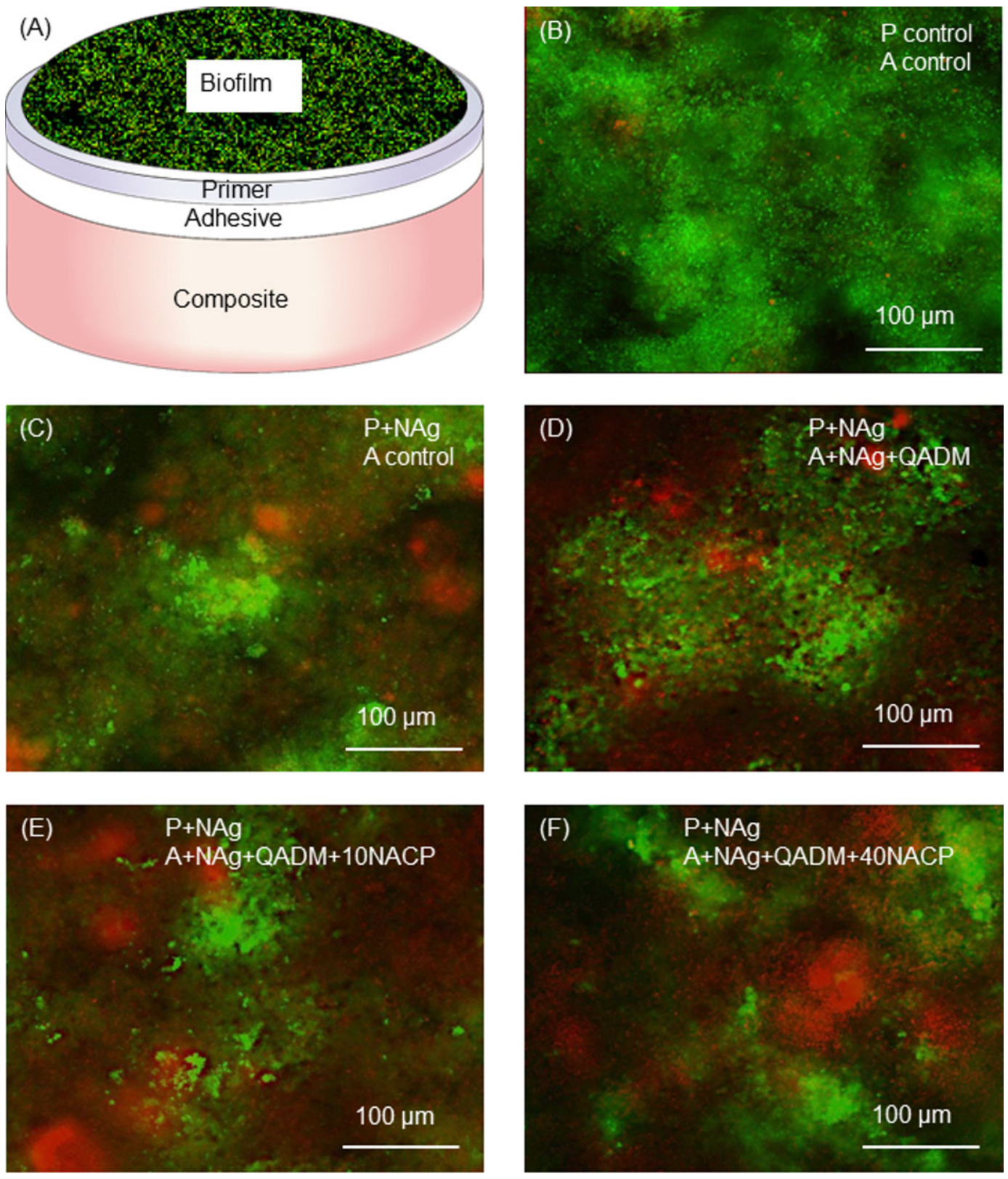

FIGURE 3. Human saliva microcosm biofilm live/dead staining assay. (A) Schematic of biofilm on cured disk with three layers: Primer, adhesive, and composite. (B-F) Representative live/dead images. Live bacteria were stained green, and dead bacteria were stained red. The live and dead bacteria in close proximity to each other produced orange/yellow colors. The biofilms were primarily alive on the control. In contrast, disks containing NAg and NAg+QADM had large amounts of dead bacteria. Incorporation of NACP into adhesive from $10 \%$ to $40 \%$ did not noticeably change the biofilm features. [Color figure can be viewed in the online issue, which is available at wileyonlinelibrary.com.]

The results showed that using the layered disks, antibacterial primer $\mathrm{P}+\mathrm{NAg}$ had strong antibacterial effects. Biofilms were inoculated on the primer layer, hence the adhesive underneath did not directly contact the biofilms. It should be noted that clinically, both antibacterial primers and adhesives are needed for caries inhibition. During a tooth cavity preparation, it is often difficult or impossible to remove all the infected and affected tissues, hence there are often residual bacteria left in the dentinal tubules of the prepared tooth cavity. ${ }^{15,22}$ This is especially true with the increased interest in less removal of tooth structure and the minimal intervention dentistry, ${ }^{54}$ which could leave behind more carious tissues with active bacteria in the tooth cavity. These residual bacteria in the tooth cavity could contribute to caries and pulp damage. Therefore, an antibacterial primer could be beneficial because the primer directly contacts the dentin and infiltrates into dentinal tubules to help disinfect the prepared tooth cavity and eradicate the residual bacteria.

In this study, the QADM was incorporated into the adhesive, while the primer contained only NAg but not QADM. This is because the addition of QADM into primer decreased the dentin bond strength in preliminary studies, while the addition of NAg into primer did not adversely affect the 


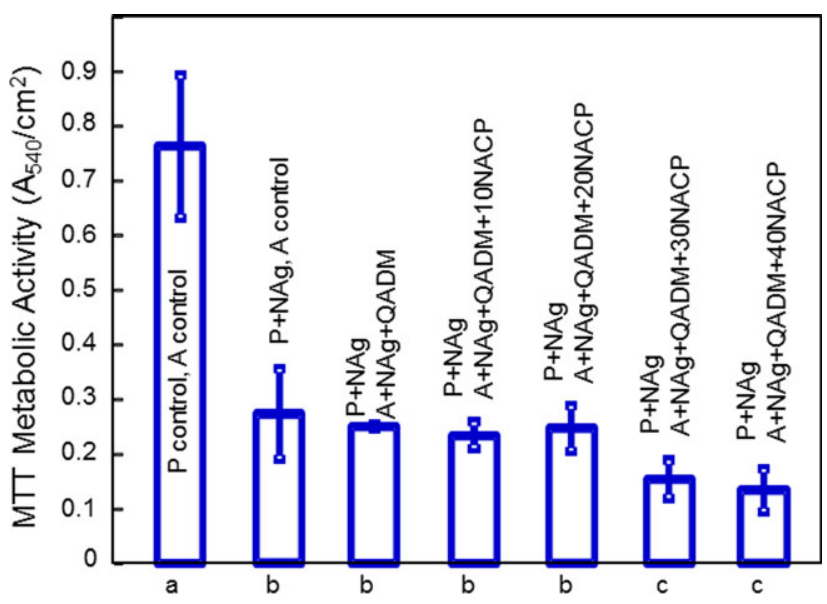

FIGURE 4. MTT metabolic activity of biofilms (mean \pm sd; $n=6$ ). The control disks had adherent biofilms with a relatively high metabolic activity. The modified bonding agents with QADM and NAg had much lower metabolic activity. The addition of NACP of $10 \%$ to $20 \%$ into the adhesive did not significantly change the metabolic activity, while $30 \%$ and $40 \%$ NACP significantly $(p<0.05)$ reduced the metabolic activity. Values with dissimilar letters at the bottom are significantly different from each other $(p<0.05)$. [Color figure can be viewed in the online issue, which is available at wileyonlinelibrary.com.]

dentin bond strength. QADM was incorporated into the adhesive. This is because an antibacterial adhesive is also beneficial clinically. Although a complete sealing of the tooth-restoration interface is highly desirable, it is difficult to achieve. Indeed, previous studies revealed microgaps between the adhesive and the primed dentin, or between the adhesive and the hybrid layer. ${ }^{55,56}$ This would suggest that a large portion of the marginal gap is surrounded by the adhesive resin, hence the invading bacteria would mostly come into contact with the adhesive surface. ${ }^{21}$ Therefore, both antibacterial primer and adhesive are needed to combat the residual bacteria, as well as the new invading bacteria along the tooth-restoration margins due to bacterial leakage, thereby to protect the pulp and inhibit recurrent caries.

The results of this study showed that incorporating $10 \%$ to $40 \%$ of NACP fillers into the BisGMA-TEGDMA adhesive did not adversely affect the dentin bond strength. The purpose of NACP incorporation was for the adhesive to obtain $\mathrm{CaP}$ ion release and remineralization capabilities. Previous studies showed that CaP-containing resins remineralized enamel and dentin lesions in vitro. ${ }^{29,30}$ Recent studies showed that NACP-containing composites released high levels of Ca and P ions. ${ }^{32}$ Furthermore, NACP nanocomposite rapidly neutralized a cariogenic acid challenge and raised the $\mathrm{pH}$ from 4 to above $6 .^{33}$ Although this study focused on the effects of NACP-containing adhesive on dentin bond strength and NACP incorporation into dentinal tubules, further study should measure the mineral content of enamel and dentin around NACP adhesive under biofilms to investigate the caries-inhibition efficacy. In addition, the results of the present showed that the incorporation of NACP had little effect on the antibacterial effects. This is because CaP materials are not known to have significant antibacterial activities. In Figure 4, a slight reduction in biofilm metabolic activity was observed at $30 \%$ and $40 \%$ NACP. In Figure 5, there was a small reduction in lactic acid production at $40 \%$ NACP. These small reductions in bacteria activity may be due to the alkaline property of NACP leading to an increase in local $\mathrm{pH}^{33}$ However, any antibacterial function from NACP appears to be minor, hence the incorporation of antibacterial agents such as QADM and NAg are needed in adhesives to achieve potent anti-biofilm capabilities.

Quaternary ammonium monomers (QAMs) have been incorporated into dental resins to obtain antibacterial functions. ${ }^{15-23,41,57}$ The QAM is copolymerized with the resin by forming a covalent bonding with the polymer network and therefore is immobilized in the resin yielding a contact-inhibition effect against bacteria that attach to the surface. ${ }^{15,16,41}$ The antibacterial mechanism of QAMs was suggested to be that, when the negatively charged bacterial cell contacts the positively charged sites of QAM resin, the electric balance of cell membrane could be disturbed, and the bacterium could explode under its own osmotic pressure. ${ }^{23,58}$ Extensive studies have been performed on MDPB-containing dental composites and bonding agents, which showed strong antibacterial effects. ${ }^{15,16,21,41}$ MDPB was effective against various oral bacteria, including facultative and obligate anaerobe in coronal lesions, and actinomyces and Candida albicans isolated from root caries. ${ }^{59-61}$ In other studies, a QAM chloride was incorporated to develop an antibacterial bonding agent. ${ }^{22}$ In addition, QAM bromides and chlorides were synthesized to develop antibacterial glass ionomer cements. ${ }^{57}$ Recently, QADM was synthesized and incorporated into composites which hindered Streptococcus mutans growth. ${ }^{20}$ Furthermore, the microcosm biofilm viability was greatly reduced when QADM was incorporated into a commercial primer. ${ }^{43}$

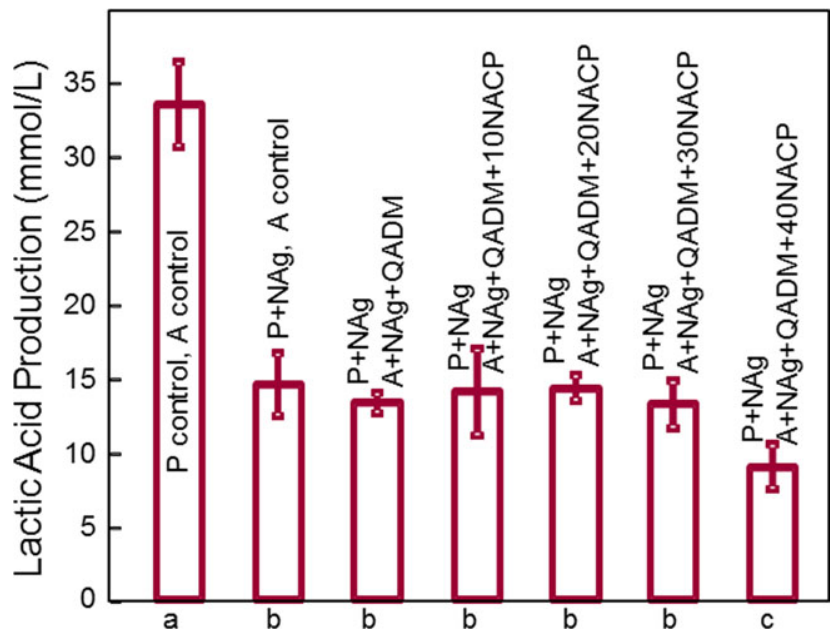

FIGURE 5. Microcosm biofilm lactic acid production (mean \pm sd; $n=$ 6). Incorporation of NAg and QADM into the bonding agent greatly lowered the lactic acid production, compared with control. NACP incorporation into the adhesive had little effect, except for the $40 \%$ NACP which significantly lowered the lactic acid production. Values with dissimilar letters at the bottom are significantly different from each other $(p<0.05)$. [Color figure can be viewed in the online issue, which is available at wileyonlinelibrary.com.] 

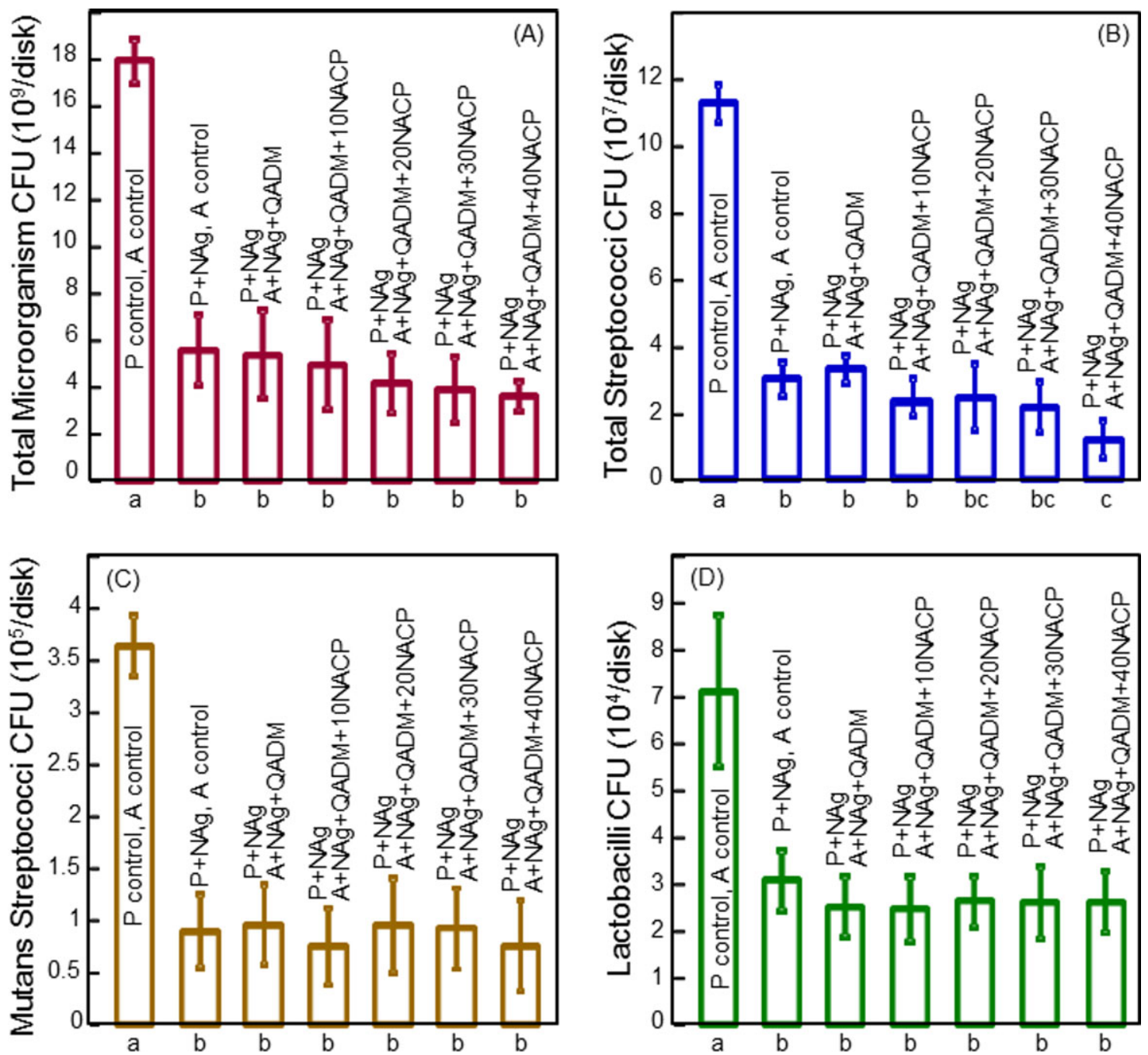

FIGURE 6. CFU counts for biofilms on the layered disks (mean $\pm s d ; n=6$ ). (A) Total microorganisms, (B) total streptococci, (C) mutans streptococci, and (D) lactobacilli. The CFU counts for biofilms adherent on the new bonding agents were reduced to about $20 \%-30 \%$ of the CFU of biofilms on the control. Hence, the new bonding agents had strong anti-biofilm effects. In each plot, values with dissimilar letters at the bottom are significantly different from each other $(p<0.05)$. [Color figure can be viewed in the online issue, which is available at wileyonlinelibrary.com.]

Besides QAMs, silver (Ag) is another effective antimicrobial agent. ${ }^{62,63}$ It was suggested that the Ag ions could inactivate the bacterial enzymes, causing the DNA to lose its replication ability, which leads to cell death. ${ }^{63} \mathrm{Ag}$ has good biocompatibility and low toxicity to human cells, has long-term antibacterial effects, ${ }^{62}$ and causes less bacterial resistance than antibiotics. ${ }^{64}$ Compared with traditional $\mathrm{Ag}$ particles of several micrometers in size, an advantage of NAg is their high surface area, so that a low filler level of NAg in resin is sufficient for the resin to be strongly antibacterial, without compromising the resin color or mechanical properties..$^{20,26,43}$ In this study, Ag salt was dissolved in TBAEMA which was then mixed with resin. Ag ions agglomerated to form nanoparticles that became part of the resin on photo-polymerization. ${ }^{20,26,43}$ An advantage of this method was that it reduced the Ag salt to NAg in situ in the resin, avoiding the mixing of preformed Ag nanoparticles which could form large agglomerates. Another advantage was that TBAEMA contained a reactive methacrylate functionality, and could be chemically incorporated into resin on photo-polymerization. In this study, NAg was incorporated into the experimental primer and adhesive, yielding potent antibacterial effects. TEM examination revealed successful incorporation of NAg and NACP into dentinal tubules. Further study should investigate the effect of NAg in the tubules on the killing of residual bacteria inside the tubules, and the effect of NACP in bonding agent on the remineralization of residual lesions in the tooth cavity.

\section{SUMMARY}

This study investigated an experimental primer comprising of PMGDM and HEMA, and an adhesive comprising of BisGMA-TEGDMA, with the incorporation of NAg, QADM and NACP for the first time. The purpose was to develop a new antibacterial primer using $\mathrm{NAg}$, and a new antibacterial adhesive using NAg and QADM, with NACP for remineralization capability. The combination of antibacterial agents (NAg and QADM) with remineralizing agent (NACP) did not 
compromise the dentin bond strength. TEM examination revealed successful incorporation of NAg and NACP into the dentinal tubules at the dentin-adhesive interface. Human saliva microcosm biofilm viability, metabolic activity, lactic acid, and CFU were greatly reduced by the new bonding agents. Therefore, the new formulations have the potential to kill residual bacteria in the tooth cavity and inhibit the invading bacteria along the tooth-restoration margins, with $\mathrm{NACP}$ for $\mathrm{Ca}$ and $\mathrm{P}$ ions to remineralize tooth lesions. The novel combination of antibacterial and remineralizing agents is promising for incorporation into a wide range of dental adhesives to inhibit caries.

\section{ACKNOWLEDGMENTS}

The authors thank Drs. Joseph M. Antonucci, Nancy J. Lin, and Sheng Lin-Gibson of the National Institute of Standards and Technology, and Dr. Gary E. Schumacher and William D. Schmuck of the Paffenbarger Research Center for discussions. They thank Esstech (Essington, PA) for donating the materials. This study was supported by NIH R01DE17974 and R01DE14190 (HX), a scholarship from the Coordination for Improvement of Higher Education/Fulbright Doctoral Program BEX0523/11-9 (MASM), National Natural Science Foundation of China grant-81100745 (LC), and the University of Maryland School of Dentistry.

\section{REFERENCES}

1. Drummond JL. Degradation, fatigue, and failure of resin dental composite materials. J Dent Res 2008;87:710-719.

2. Ferracane JL. Resin composite-State of the art. Dent Mater 2011; 27:29-38.

3. Demarco FF, Correa MB, Cenci MS, Moraes RR, Opdam NJM. Longevity of posterior composite restorations: Not only a matter of materials. Dent Mater 2012;28:87-101.

4. Bayne SC, Thompson JY, Swift EJ Jr, Stamatiades P, Wilkerson M. A characterization of first-generation flowable composites. J Am Dent Assoc 1998;129:567-577.

5. Lim BS, Ferracane JL, Sakaguchi RL, Condon JR. Reduction of polymerization contraction stress for dental composites by twostep light-activation. Dent Mater 2002;18:436-444.

6. Watts DC, Marouf AS, Al-Hindi AM. Photo-polymerization shrinkage-stress kinetics in resin-composites: Methods development. Dent Mater 2003;19:1-11.

7. Samuel SP, Li S, Mukherjee I, Guo Y, Patel AC, Baran GR, Wei Y. Mechanical properties of experimental dental composites containing a combination of mesoporous and nonporous spherical silica as fillers. Dent Mater 2009;25:296-301.

8. Zalkind MM, Keisar O, Ever-Hadani P, Grinberg R, Sela MN. Accumulation of Streptococcus mutans on light-cured composites and amalgam: An in vitro study. J Esthet Dent 1998;10:187-190.

9. Beyth N, Domb AJ, Weiss El. An in vitro quantitative antibacterial analysis of amalgam and composite resins. J Dent 2007;35: 201-206.

10. Featherstone JD. The continuum of dental caries-Evidence for a dynamic disease process. J Dent Res 2004;83:C39-C42.

11. ten Cate JM. Biofilms, a new approach to the microbiology of dental plaque. Odontology 2006;94:1-9.

12. Deligeorgi V, Mjor IA, Wilson NH. An overview of reasons for the placement and replacement of restorations. Prim Dent Care 2001; 8:5-11.

13. Jokstad A, Bayne S, Blunck U, Tyas M, Wilson N. Quality of dental restorations. FDI Commision Projects 2-95. Int Dent J 2001;51: 117-158.

14. NIDCR (National Institute of Dental and Craniofacial Research) announcement \# 13-DE-102, Dental Resin Composites and Caries, Bethesda, MD, USA, March 5, 2009.
15. Imazato S, Kinomoto $\mathrm{Y}$, Tarumi H, Ebisu S, Tay FR. Antibacterial activity and bonding characteristics of an adhesive resin containing antibacterial monomer MDPB. Dent Mater 2003;19: 313-319.

16. Imazato S. Bioactive restorative materials with antibacterial effects: New dimension of innovation in restorative dentistry. Dent Mater J 2009;28:11-19.

17. Xu X, Wang Y, Liao S, Wen ZT, Fan Y. Synthesis and characterization of antibacterial dental monomers and composites. J Biomed Mater Res B 2012;100:1511-1162.

18. Weng Y, Howard L, Guo X, Chong VJ, Gregory RL, Xie D. A novel antibacterial resin composite for improved dental restoratives. J Mater Sci Mater Med 2012;23:1553-1561.

19. Antonucci JM, Zeiger DN, Tang K, Lin-Gibson S, Fowler BO, Lin NJ. Synthesis and characterization of dimethacrylates containing quaternary ammonium functionalities for dental applications. Dent Mater 2012;28:219-228.

20. Cheng L, Weir MD, Xu HHK, Antonucci JM, Kraigsley AM, Lin NJ, Lin-Gibson S, Zhou XD. Antibacterial amorphous calcium phosphate nanocomposite with quaternary ammonium salt and silver nanoparticles. Dent Mater 2012;28:561-572.

21. Imazato S, Tay FR, Kaneshiro AV, Takahashi Y, Ebisu S. An in vivo evaluation of bonding ability of comprehensive antibacterial adhesive system incorporating MDPB. Dent Mater 2007;23: 170-176.

22. Li F, Chen J, Chai Z, Zhang L, Xiao Y, Fang M, Ma S. Effects of a dental adhesive incorporating antibacterial monomer on the growth, adherence and membrane integrity of Streptococcus mutans. J Dent 2009;37:289-296.

23. Namba N, Yoshida $Y$, Nagaoka N, Takashima S, Matsuura-Yoshimoto K, Maeda H, Van Meerbeek B, Suzuki K, Takashida S. Antibacterial effect of bactericide immobilized in resin matrix. Dent Mater 2009;25:424-430.

24. Morones JR, Elechiguerra JL, Camacho A, Holt K, Kouri JB, Ramirez JT, Yacaman MJ. The bactericidal effect of silver nanoparticles. Nanotechnology 2005;16:2346-2353.

25. Fan C, Chu L, Rawls HR, Norling BK, Cardenas HL, Whang K. Development of an antimicrobial resin-A pilot study. Dent Mater 2011;27:322-328.

26. Cheng YJ, Zeiger DN, Howarter JA, Zhang X, Lin NJ, Antonucci JM, Lin-Gibson S. In situ formation of silver nanoparticles in photocrosslinking polymers. J Biomed Mater Res 2011;97B: 124-131.

27. Allaker RP. The use of nanoparticles to control oral biofilm formation. J Dent Res 2010;89:1175-1186.

28. Skrtic D, Antonucci JM, Eanes ED, Eichmiller FC, Schumacher GE. Physiological evaluation of bioactive polymeric composites based on hybrid amorphous calcium phosphates. J Biomed Mater Res B 2000;53:381-391.

29. Dickens SH, Flaim GM, Takagi S. Mechanical properties and biochemical activity of remineralizing resin-based $\mathrm{Ca}-\mathrm{PO}_{4}$ cements. Dent Mater 2003;19:558-566.

30. Langhorst SE, O'Donnell JNR, Skrtic D. In vitro remineralization of enamel by polymeric amorphous calcium phosphate composite: Quantitative microradiographic study. Dent Mater 2009;25: 884-891.

31. Xu HHK, Weir MD, Sun L, Moreau JL, Takagi S, Chow LC, Antonucci JM. Strong nanocomposites with $\mathrm{Ca}, \mathrm{PO}_{4}$ and $\mathrm{F}$ release for caries inhibition. J Dent Res 2010;89:19-28.

32. Xu HHK, Moreau JL, Sun L, Chow LC. Nanocomposite containing amorphous calcium phosphate nanoparticles for caries inhibition. Dent Mater 2011;27:762-769.

33. Moreau JL, Sun L, Chow LC, Xu HHK. Mechanical and acid neutralizing properties and inhibition of bacterial growth of amorphous calcium phosphate dental nanocomposite. J Biomed Mater Res 2011;98B:80-88.

34. Spencer $P$, Wang $Y$. Adhesive phase separation at the dentin interface under wet bonding conditions. J Biomed Mater Res 2002;62:447-456.

35. Ikemura K, Tay FR, Endo T, Pashley DH. A review of chemicalapproach and ultramorphological studies on the development of fluoride-releasing dental adhesives comprising new pre-reacted glass ionomer (PRG) fillers. Dent Mater J 2008;27:315-329. 
36. Ritter AV, Swift EJ Jr, Heymann HO, Sturdevant JR, Wilder AD Jr. An eight-year clinical evaluation of filled and unfilled one-bottle dental adhesives. J Am Dent Assoc 2009;140:28-37.

37. Park J, Eslick J, Ye Q, Misra A, Spencer P. The influence of chemical structure on the properties in methacrylate-based dentin adhesives. Dent Mater 2011;27:1086-1093.

38. Pashley DH, Tay FR, Breschi L, Tjaderhane L, Carvalho RM, Carrilho M, Tezvergil-Mutluay A. State of the art etch-and-rinse adhesives. Dent Mater 2011;27:1-16.

39. Spencer P, Ye Q, Park JG, Topp EM, Misra A, Marangos O, Wang Y, Bohaty BS, Singh V, Sene F, Eslick J, Camarda K, Katz JL. Adhesive/dentin interface: The weak link in the composite restoration. Ann Biomed Eng 2010;38:1989-2003.

40. Van Meerbeek B, Yoshihara K, Yoshida Y, Mine A, De Munck J. State of the art of self-etch adhesives. Dent Mater 2011;27:17-28.

41. Imazato S, Ehara A, Torii M, Ebisu S. Antibacterial activity of dentine primer containing MDPB after curing. J Dent 1998;26: 267-271.

42. Hiraishi N, Yiu CK, King NM, Tay FR. Effect of chlorhexidine incorporation into a self-etching primer on dentine bond strength of a luting cement. J Dent 2010;38:496-502.

43. Cheng L, Zhang K, Melo MAS, Weir MD, Zhou XD, Xu HHK. Anti-biofilm dentin primer with quaternary ammonium and silver nanoparticles. J Dent Res 2012;91:598-604.

44. Dickens $\mathrm{SH}, \mathrm{Cho} \mathrm{BH}$. Interpretation of bond failure through conversion and residual solvent measurements and Weibul analyses of flexural and microtensile bond strengths of bonding agents. Dent Mater 2005;21:354-364.

45. Sadek FT, Mazzoni A, Breschi L, Tay FR, Braga RR. Six-month evaluation of adhesives interface created by a hydrophobic adhesive to acid-etched ethanol-wet bonded dentine with simplified dehydration protocols. J Dent 2010;38:276-283.

46. Chow LC, Sun L, Hockey B. Properties of nanostructured hydroxyapatite prepared by a spray drying technique. J Res NIST 2004 109:543-551.

47. Antonucci JM, O'Donnell JN, Schumacher GE, Skrtic D. Amorphous calcium phosphate composites and their effect on composite-adhesive-dentin bonding. J Adhes Sci Technol 2009;23: 1133-1147.

48. Van Meerbeek B, Yoshida $Y$, Lambrechts $P$, Vanherle G, Duke ES Eick JD, Robinson SJ. A TEM study of two water-based adhesive systems bonded to dry and wet dentin. J Dent Res 1998;77:50-59.

49. McBain AJ. In vitro biofilm models: An overview. Adv Appl Microbiol 2009;69:99-132.

50. McBain AJ, Sissons C, Ledder RG, Sreenivasan PK, De Vizio W, Gilbert P. Development and characterization of a simple perfused oral microcosm. J Appl Microbiol 2005;98:624-634.
51. Lima JP, Sampaio de Melo MA, Borges FM, Teixeira AH, SteinerOliveira C, Nobre Dos Santos M, Rodrigues LK, Zanin IC. Evaluation of the antimicrobial effect of photodynamic antimicrobial therapy in an in situ model of dentine caries. Eur J Oral Sci 2009; 117:568-574.

52. Hildebrandt GH, Bretz WA. Comparison of culture media and chairside assays for enumerating mutans streptococci. J Appl Microbiol 2006;100:1339-1347.

53. Park JH, Tanabe $Y$, Tinanoff N, Turng BF, Lilli H, Minah GE. Evaluation of microbiological screening systems using dental plaque specimens from young children aged 6-36 months. Caries Res 2006;40:277-280.

54. Murdoch-Kinch CA, McLean ME. Minimally invasive dentistry. J Am Dent Assoc 2003;134:87-95.

55. Duarte SJ, Lolato $A L$, de Freitas $C R$, Dinelli W. SEM analysis of internal adaptation of adhesive restorations after contamination with saliva. J Adhes Dent 2005;7:51-56.

56. Loguercio AD, Reis A, Bortoli G, Patzlaft R, Kenshima S, Rodrigues Filho LE, Accorinte Mde L, van Dijken JW. Influence of adhesive systems on interfacial dentin gap formation in vitro. Oper Dent 2006;31:431-441.

57. Xie D, Weng Y, Guo X, Zhao J, Gregory RL, Zheng C. Preparation and evaluation of a novel glass-ionomer cement with antibacterial functions. Dent Mater 2011;27:487-496.

58. Beyth N, Yudovin-Farber I, Bahir R, Domb AJ, Weiss El. Antibacterial activity of dental composites containing quaternary ammonium polyethylenimine nanoparticles against Streptococcus mutans. Biomaterials 2006;27:3995-4002.

59. Imazato S, Ebi N, Tarumi H, Russell RRB, Kaneko T, Ebisu S. Bactericidal activity and cytotoxicity of antibacterial monomer MDPB. Biomaterials 1999;20:899-903.

60. Imazato S, Torii Y, Takatsuka T, Inoue K, Ebi N, Ebisu S. Bactericidal effect of dentin primer containing antibacterial monomer methacryloyloxydodecylpyridinium bromide (MDPB) against bacteria in human carious dentin. J Oral Rehabil 2001;28:314-319.

61. Yoshikawa K, Clark DT, Brailsford SR, Beighton D, Watson TF, Imazato S, Momoi Y. The effect of antibacterial monomer MDPB on the growth of organisms associated with root caries. Dent Mater J 2007;26:388-392.

62. Damm C, Munsted H, Rosch A. Long-term antimicrobial polyamide 6/silver nanocomposites. J Mater Sci 2007;42:6067-6073.

63. Rai M, Yada A, Gade A. Silver nanoparticles as a new generation of antimicrobials. Biotechnol Adv 2009;27:76-83.

64. Percival SL, Bowler PG, Russell D. Bacterial resistance to silver in wound care. J Hosp Infect 2005;60:1-7. 